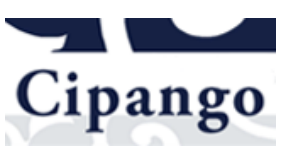

\title{
Cipango
}

Cahiers d'études japonaises

16 | 2009

L'invention des « arts populaires » - Yanagi Sōetsu et le Mingei

\section{Et le sourire de Mokujiki révéla à Yanagi Sōetsu la beauté véritable}

And Mokujiki's smile revealed true beauty to Yanagi Sōetsu

\section{François Macé}

\section{(2) OpenEdition}

\section{Journals}

Édition électronique

URL : https://journals.openedition.org/cipango/370

DOI : $10.4000 /$ cipango.370

ISSN : 2260-7706

Éditeur

INALCO

Édition imprimée

Date de publication : 1 janvier 2009

Pagination : 73-87

ISSN : 1164-5857

\section{Référence électronique}

François Macé, «Et le sourire de Mokujiki révéla à Yanagi Sōetsu la beauté véritable », Cipango [En ligne], 16 | 2009, mis en ligne le 15 novembre 2011, consulté le 30 juin 2021. URL : http:// journals.openedition.org/cipango/370; DOI : https://doi.org/10.4000/cipango.370

Ce document a été généré automatiquement le 30 juin 2021.

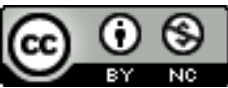

Cipango est mis à disposition selon les termes de la Licence Creative Commons Attribution - Pas d'Utilisation Commerciale 4.0 International. 


\title{
Et le sourire de Mokujiki révéla à Yanagi Sōetsu la beauté véritable
}

\author{
And Mokujiki's smile revealed true beauty to Yanagi Sōetsu
}

\section{François Macé}

1 Pour beaucoup d'amateurs d'art japonais, les noms de Mokujiki 木喰 (1718-1810) et d'Enkū 円空 (1632-1695) sont indissociables malgré les cent ans qui les séparent. Pour ma part, ils restent liés dans mon esprit à l'exposition Enkū Mokujiki ten, à Tōkyō, en 1972, où leurs œuvres étaient présentées ensemble. À cette époque, ils représentaient les deux figures majeures d'un art bouddhique plein de vigueur. Ils apportaient la démonstration de la vitalité des artistes japonais pendant l'époque d'Edo, loin de la prétendue dégénérescence de l'art bouddhique aux Temps modernes.

2 Pourtant ces deux moines sculpteurs, qui paraissent aussi inséparables que Kanzan 寒 山 et Jittoku 拾得, n'ont été associés qu'assez récemment, au moment du second boom de Mokujiki. La première exposition commune s'est tenue en 1953 à Hokkaidō ${ }^{1}$. Ils ont longtemps été ignorés des spécialistes de l'histoire de l'art. Considérées comme trop grossières, leurs sculptures n'entraient pas dans les cadres d'un art synonyme de haute culture. Ils ne répondaient tout simplement pas aux critères du beau. L'un paraissait trop sauvage et violent, l'autre trop naïf et maladroit.

Mais avant même de les mettre en parallèle, il fallait d'abord les connaître. Ce fut le travail de Yanagi Sōetsu, qui sortit Mokujiki de l'oubli. Car Enkū, lui, n'avait pas disparu des mémoires.

\section{Enkū}

Enkū était en effet connu depuis longtemps. J'en veux pour preuve la place qu'il occupe dans des ouvrages de référence des années vingt et trente $\mathrm{du} \mathrm{xx}^{\mathrm{e}}$ siècle. Dans le Grand dictionnaire du bouddhisme, dont la première édition date de 1933², Enkū a droit à une notice avec son portrait. On y apprend qu'il bénéficiait d'une biographie dans le livre XI de la Suite de la Biographie des moines illustres ${ }^{3}$. Il est présenté comme un moine peintre et 
sculpteur n'utilisant qu'une serpe et ayant voyagé dans le Japon depuis Kyūshū jusqu'aux terres des Ezo (Aïnu) qu'il était allé prêcher. Il vivait d'ordinaire dans des cavernes. Le dictionnaire ne fait aucun commentaire sur la qualité artistique de ses œuvres, mais note leur efficace pour pacifier des esprits dangereux. Il insiste sur la sainteté du personnage, qualifié de «Shakya actuel » (Ima Shaka 今釈迦).

Or, si la Suite de la Biographie des moines illustres cite Enkū, c'est qu'il n'avait jamais été oublié. À peine vingt ans après sa mort, la célèbre Description illustrée des trois entités de l'univers au Japon et en Chine $e^{4}$ notait qu'Enkū avait offert mille Jizō au mont Osorezan 恐 $山^{5}$. Un peu plus tard, le moine bénéficia encore d'une notice dans la Biographie des personnes étranges des temps récents parue en $1790^{6}$. Une planche l'y représente même en train de sculpter un Bouddha sur un arbre vivant. Cet ouvrage fut suivi à intervalles réguliers d'autres publications retraçant la vie d'Enkū.

6 La Grande Encyclopédie de Heibonsha, parue elle entre 1931 et 1933, lui consacra une notice de la même importance que le Grand dictionnaire du bouddhisme, avec le même portrait ${ }^{7}$. Elle précisait qu'Enkū était un moine de l'école Rinzai (alors qu'il mena en réalité une vie de moine sans attache, proche de celle des yamabushi, les ascètes des montagnes). Ces renseignements plus ou moins sûrs semblent provenir de la même source, probablement la Suite de la Biographie des moines illustres. Très curieusement ces deux dictionnaires ne mentionnent aucune date. L'Encyclopédie situe même le voyage d'Enkū chez les Ezo à la fin de la période Ashikaga ( $\mathrm{xvI}^{\mathrm{e}} \mathrm{s}$.). Les commentateurs insistent surtout sur la piété qui l'avait poussé à produire de nombreuses statues, sans jamais évoquer la valeur de celles-ci.

7 Cette valeur ne sera reconnue que fort tard, et après celle de Mokujiki. Cela commencera avec le sculpteur Hashimoto Heihachi 橋本平八 (1897-1935), qui découvre une œuvre d'Enkū lors d'un séjour au temple Senkōji 千光寺, au retour d'un voyage à Takayama, en 1931. Il décide immédiatement d'étudier ces sculptures et leur auteur. Ce travail lui prendra dix ans. Au moment de sa découverte, il écrit dans son journal ${ }^{8}$ :

Je ne sais quel était l'état d'esprit de Yanagi Sōetsu quand il étudia le vénérable Mokujiki, mais aujourd'hui, quand je pense à Enkū en contemplant ses ceuvres, je peux le ressentir.

8 Il rapporte également sa réaction, qui reprend celle de Yanagi devant les premières œuvres de Mokujiki :

Le saint homme [Enkū] a produit d'innombrables bouddhas au Senkōji, il y a maintenant plus de deux cent trente ans. Il est pourtant difficile de croire que cela remonte à si loin. [Ces bouddhas] donnent l'impression que nous n'en sommes séparés que d'un laps de temps très court, tout au plus quelques années.

Pourtant, malgré la passion que lui voue Hashimoto, la renommée d'Enkū ne sera assurée que dans les années 1960, après une première exposition particulière en 1957, à Kamakura. La revue Mingei avait pris les devants en lui consacrant, en 1959, un numéro spécial qui comprenait un article de Yanagi: "Liens karmiques avec les bouddhas d'Enkū »'. Comme le montre le titre du volume de ses œuvres choisies dans lequel cet article fut par la suite inséré, Le vénérable Mokujiki, Yanagi s'intéressa en effet à Enkū à travers son expérience de Mokujiki ${ }^{10}$.

\section{Un oublié}

10 Les deux dictionnaires des années 1930 s'accordent aussi pour ignorer Mokujiki. Mochizuki ne mentionne même aucun personnage de ce nom, alors que le bouddhisme 
japonais en a connu un certain nombre. L'Encyclopédie cite elle une personne, mais il s'agit du fameux Mokujiki Ōgo 木食應其 (1536-1608), moine du Kōyasan, contemporain de Toyotomi Hideyoshi, qui fraya avec les puissants de son temps, et laissa bien des poèmes (renga), mais pas la moindre sculpture.

Le sculpteur Mokujiki, Mokujiki Gogyō 木喰五行, avait bel et bien disparu. Lors de ses recherches dans la province natale du moine, Yanagi n'a retrouvé sa trace dans aucune des histoires régionales de son époque. Il évoque par exemple la très détaillée Monographie de la province de Kai ${ }^{11}$, qui ignore complètement Mokujiki. Autrement dit, peu de temps après sa mort, Mokujiki était déjà oublié. On peut supposer que de son vivant même, sa renommée devait être limitée.

Son parcours ressemble pourtant beaucoup à celui d'Enkū dont le souvenir était resté vivant. Tous les deux sont des «moines vagabonds » (yugyōsō 遊行僧) qui pendant une grande partie de leur vie vécurent en marge des institutions. Tous les deux parcourent tout le Japon, de Kyūshū à Ezo. Tous les deux, enfin, produisirent une quantité étonnante de sculptures. Cette activité, depuis longtemps considérée comme une œuvre pie dans le bouddhisme, faisait partie intégrante de leur pratique, à côté d'autres ascèses, comme de ne pas loger dans un bâtiment mais dans des grottes pour Enkū, ou de ne se nourrir uniquement de fruits d'arbre comme Mokujiki, ainsi que l'indique son nom $^{12}$. Si les moines vagabonds ont été nombreux, les saints hommes capables de se fondre dans la foule et, paradoxalement, de laisser un nom ont dû l'être beaucoup moins. Ryōkan 良寛 (1758-1831) connut de son vivant une certaine notoriété. Tōsui Unkei 桃水雲, qui vécut au XVII ${ }^{\mathrm{e}}$ siècle, finit sa vie comme marchand de vinaigre, mais a les honneurs de la Biographie des personnes étranges des temps récents ${ }^{13}$. Quant aux sculpteurs de la carrure de Mokujiki, il est probable qu'ils furent encore plus rares. On connaît un autre Mokujiki, Mokujiki Sankyo 木喰山居 (1657-1724), dont on dit qu'il sculpta dix mille bouddhas ${ }^{14}$. Et l'on retrouve Enkū. Gorai Shigeru suppose d'ailleurs que c'est la découverte des statues d'Enkū à Hokkaidō qui poussa Mokujiki à suivre ses traces ${ }^{15}$.

\section{La découverte}

La redécouverte de Mokujiki paraît fortuite. Yanagi Sōetsu raconte que le 9 janvier 1923, il s'était rendu chez un certain Komiyama Seizō 小宮山清三, maire du village d'Ikeda dans le département de Yamanashi. Il était accompagné d'Asakawa Takumi 浅 川巧 (1891-1931), qui s'était spécialisé dans l'artisanat coréen. Tous deux voulaient voir les porcelaines coréennes que Komiyama collectionnait. Yanagi, âgé de 34 ans, travaillait alors au projet de Musée des arts populaires coréens (Chōsen minzoku bijutsukan 朝鮮民族美術館) ${ }^{16}$. Dans la réserve de son hôte se trouvaient, outre les poteries qu'il venait voir, deux sculptures qui attirèrent immédiatement son regard. Il s'agissait d'une statue du bodhisattva Ksitigarbha, Jizō bosatsu 地蔵菩薩, ainsi que d'une autre du tathâgata Amitâyus, Muryōju nyorai 無量寿如来. 


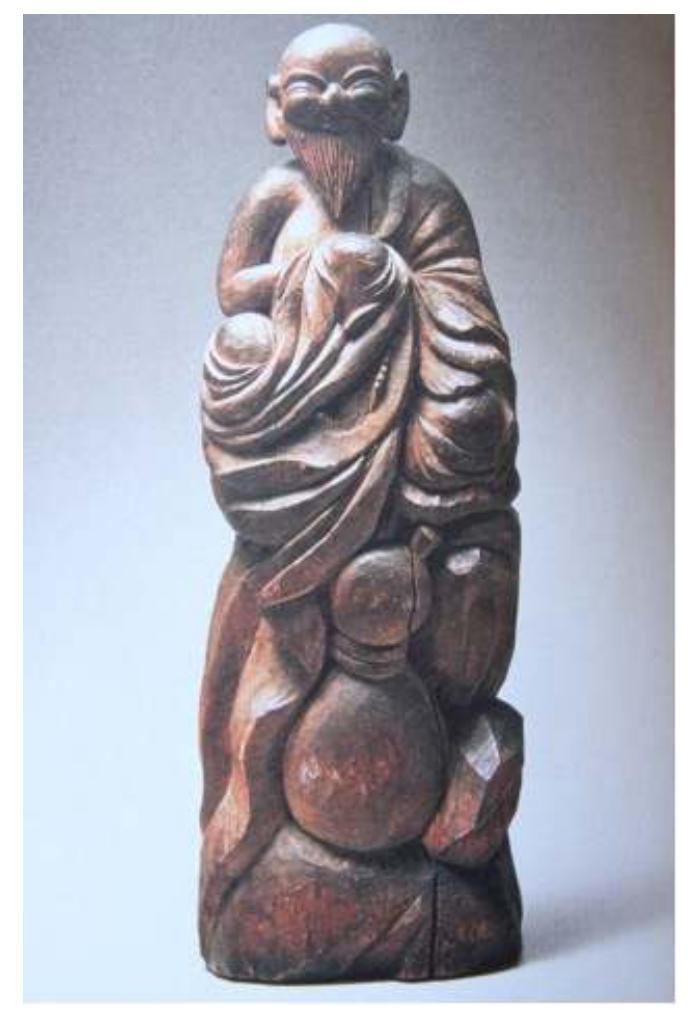

MOKUJIKI, BODHISATTVA KSITIgARBHA (JIZô BOSATSU 地蔵菩薩) MINgEIKAN, TôKYô

Une dernière statue, celle de Namu daishi 南無大師 ${ }^{17}$ se trouvait dans la pièce de réception. Toutes portaient le nom d'un certain Mokujiki. L'enthousiasme de Yanagi devant la beauté de ces œuvres fut tel que Komiyama lui offrit le Jizō et l'aida par la suite dans sa quête du mystérieux sculpteur Mokujiki.

Le soir même de sa découverte, Yanagi écrivit à un de ses amis sculpteur que son intuition ne le trompait pas, le Saint homme (Mokujiki) était le plus grand sculpteur de la fin du bakufu (première moitié du XIX siècle). Rentré chez lui, malade, il avait déposé le Jizō près de lui. Celui-ci veilla à son chevet. « À partir de ce moment-là, chaque jour, chaque soir, je vécus avec ce bouddha $»$ rapporte-t-i ${ }^{18}$.

Ce n'est pas tout à fait un hasard si ces statues se trouvaient à Ikeda. Mokujiki était luimême né pas très loin, dans l'actuel département de Yamanashi. En outre, au cours de ses longs périples dans tout le Japon, il était revenu trois fois dans son village natal de Marubatake 丸畑. La dernière, à l'âge de 83 ans, fut la plus productive. Sur la demande d'un des villageois, il entreprit de construire un Pavillon de Shikoku, Shikokudō 四国 堂, qui permettait d'accomplir à moindre effort le fameux pèlerinage en quatre-vingthuit étapes (Hachijūhakkasho八十八力所) du tour de Shikoku ${ }^{19}$. Les quatre-vingt-onze sculptures exécutées par Mokujiki pour ce pavillon permirent la découverte. En effet, le bâtiment fut détruit à l'époque de Taishō (1912-1926), et les statues qu'il contenait, dispersées peu de temps avant le passage de Yanagi. Ce sont trois d'entre elles qu'il vit chez Komiyama. Le souvenir de leur provenance n'avait pas eu le temps d'être effacé.

Il faut noter que ces sculptures appartiennent à l'époque où la maîtrise du moine était à son apogée. Yanagi aurait-il vu des statues de la première période de Mokujiki qu'il n'aurait peut-être pas été saisi de la même façon? 


\section{La recherche}

18 Si Yanagi put peu à peu découvrir qui était ce moine, c'est que ce dernier avait inscrit au dos des statues outre son nom, divers autres renseignements qui permirent de retrouver ses traces. Il inscrivait souvent, en particulier, la formule suivante ${ }^{20}$ :

Parcourant tout le Japon, considérant les huit écoles d'un seul regard, ne pensant qu'à l'édification [de tous], le sculpteur de Bouddha les dépose dans les endroits avec lesquels il a une attache dans les diverses provinces. C'est son vœu fondamental parmi les dix grands vœu $x^{21}$. Toutes font partie des mille statues du Japon.

日本順国八宗一見之行想，十大願之内本願として仏を仏師国々因縁ある所に これをほどこす、みな千躰之内なり

Ce texte était accompagné de la date de l'« ouverture des yeux» de la statue et du nom du sculpteur.

Le second temps de la quête de Yanagi fut marqué par la découverte des papiers de Mokujiki dans son village natal. Sa famille avait conservé ses carnets consignant les lieux où il avait été hébergé22 et ceux dans lesquels il avait effectué des offrandes de sutras $^{23}$, un recueil de poèmes (waka), et surtout ce qui apparaît comme son autobiographie, le Miroir de mes vœux intimes du Pavillon de Shikoku (Shikokudō Shingankyō 四国堂心願鏡). Yanagi raconte sa joie de pouvoir consulter ces documents. Il recopiera fébrilement le Miroir de mes vœux intimes en une nuit, car il lui avait été demandé de le rendre dès le lendemain. Grâce à l'intervention de Komiyama, il put toutefois emprunter ces documents par la suite et les étudier à loisir.

Grâce à ces données, son enquête put être menée avec une certaine précision ${ }^{24}$. Yanagi se lança rapidement sur les traces de Mokujiki. En moins d'un an, il réussit à retrouver trois cents statues un peu partout au Japon. Il mit par ailleurs à contribution ses amis, tel Mushanokōji Saneatsu 武者小路実篤 $(1885-1976)^{25}$, dont Yanagi profita de l'installation à Kyūshū où il avait fondé une sorte de phalanstère, pour lui demander de rechercher les statues de Mokujiki qu'il savait se trouver sur l'île. Yanagi publia ses premiers résultats en 1924-1925 dans sept livraisons de la revue Josei 女性 (Femme), avant de les rassembler la même année en un volume, intitulé Mokujiki shōnin no kenkyū 木喰上人之研究 (Études sur le vénérable Mokujiki) ${ }^{26}$. Il poursuivit ses recherches jusqu'en 1926, avant de se consacrer entièrement au mouvement des Arts populaires. L'impulsion avait été donnée. D'autres chercheurs pourront poursuivre sa tâche, soutenus par le Groupe d'études mokujikiennes (Mokujiki kenkyūkai), qu'il avait créé. Yanagi organisa encore la première exposition à Kyōto consacrée à Mokujiki, en 1935.

\section{La beauté authentique}

Yanagi n'est pas un historien de métier. C'est l'œuvre et la personnalité de Mokujiki qui l'intéresse. Dans le livre qu'il consacre au moine sculpteur, il explique que le hasard qui lui fit découvrir Mokujiki n'a pu jouer que parce que trois conditions préalables avaient été remplies. La première est sa propre recherche de la «beauté véritable " (shin no bi 真の美). Jamais il n'avait pu vivre séparé du monde de la beauté. C'est ce qui l'avait amené à étudier William Blake quand il était étudiant, puis la porcelaine coréenne de la dynastie Yi. C'est cette beauté véritable qu'il reconnaît dans les sculptures de Mokujiki. Yanagi revient sur cette beauté vers la fin de son ouvrage, quand il traite des statues du 
Pavillon de Shikoku. Pour lui, Mokujiki n'a jamais cherché à créer de beaux objets. Pourtant, tout le monde admet qu'il a créé des œuvres originales. Car il a su se libérer des formes fixes. Yanagi convient certes ${ }^{27}$ :

D'un certain point de vue, ses créations peuvent être considérées comme laides [...]. Mais pour lui qui était devenu un religieux libre d'attache, pourquoi aurait-il pu y avoir une hésitation vers la laideur? À plus forte raison, où y a-t-il une opposition à la beauté? Pour tout, il est sans pensée [mushin 無心]. L'absence d'apprêt dans la beauté ne signifie pas qu'il manque de la beauté. La laideur apparente ne vient pas de ce qu'il y a laideur. Les hommes modernes ont désiré saisir une nouvelle beauté à partir de la laideur. On pourrait dire que les œuvres du saint homme sont modernes. Mais à l'inverse de l'époque actuelle, qui est une époque d'opposition, l'esprit du Saint homme avait atteint le point où beauté et laideur ne sont plus distinctes [bishū funi 美醜不二]. En effet, si l'on choisit le beau contre le laid, c'est encore une passion obscurcissante.

Il continue en prenant le parti de la simplicité :

simplicité ne veut pas dire grossièreté, sans pensée [mushin 無心] ne signifie pas ignorance. Aussi l'art de la foi montre-t-il d'ordinaire une beauté simple. Une réflexion artificielle ne produit pas de l'art. Son art est simple, il est naturel [...]. Il avait conscience de ne pas connaître la technique et n'en avait pas honte.

Cette beauté simple s'exprime dans le sourire, sourire de Mokujiki, sourire des Bouddhas :

Ce qui frappe, qui que l'on soit, c'est l'expression de son visage. Quel sculpteur a pu comme lui saisir et rendre si profondément le sourire dans le corps des bouddhas? Dans l'histoire de la sculpture, les bouddhas souriants commencent avec lui (...). Par ce sourire les Bouddhas nous deviennent familiers.

\section{Un art populaire}

La deuxième condition préalable à sa rencontre avec Mokujiki concerne le caractère populaire des sculptures. Yanagi insiste sur leur côté provincial, villageois, paysan. Il les compare aux poteries des anonymes (getemono 下手物) méprisées des collectionneurs amateurs de signatures célèbres. Lui a immédiatement perçu leur étonnante beauté cachée. Tout au long de ses écrits sur Mokujiki, il insiste sur le caractère non apprêté des œuvres. Selon lui, Mokujiki n'a jamais eu de maître. Il n'appartient à aucune école. Il n'a jamais recherché la gloire et le renom. S'il a laissé son nom derrière les statues, il a déposé celles-ci dans des endroits isolés en pleine campagne, et non pas dans des lieux célèbres et visités ${ }^{28}$.

Le peuple, c'est l'ami que s'est choisi le saint homme [...]. Il a déposé ses statues dans des chapelles au bord des chemins [...]. Les Bouddhas sont sortis des temples et marchent dans les villes et les villages ${ }^{29}$.

C'est la combinaison entre la simplicité des moyens et une profondeur non préméditée qui attire Yanagi. Ce sera son fil conducteur dans le mouvement pour les Arts populaires. Son intérêt pour les images d'ōtsu répond à la même logique ${ }^{30}$.

Mais ce caractère populaire ne signifie pas que Mokujiki était ignorant, loin de là. Moine de l'école ésotérique Shingon, il connaissait les lettres germes ${ }^{31}$ de tous les personnages du panthéon bouddhique qu'il sculpta, une cinquantaine de noms selon les comptes de Yanagi ${ }^{32}$. Il laissa aussi comme nous l'avons vu un recueil de poèmes.

La personnalité de Mokujiki pose un autre problème. Ce moine vagabond, qui n'a guère retenu l'attention de ses contemporains, n'était pas un artisan anonyme. Non seulement il signait ses œuvres, mais de plus il s'attribuait des titres extraordinaires : 
Bodhisattva Gogyō (Gogyō bosatsu), Immortel Myōman (Myōman sennin)... La réalisation de son vœu de faire le tour du Japon en offrant des milliers de Bouddha l'avait fait accéder à un statut dépassant la simple humanité. De la même façon qu'il sculpta bouddhas et bodhisattvas, il se représenta lui-même d'assez nombreuses fois.

\section{Autoportrait}

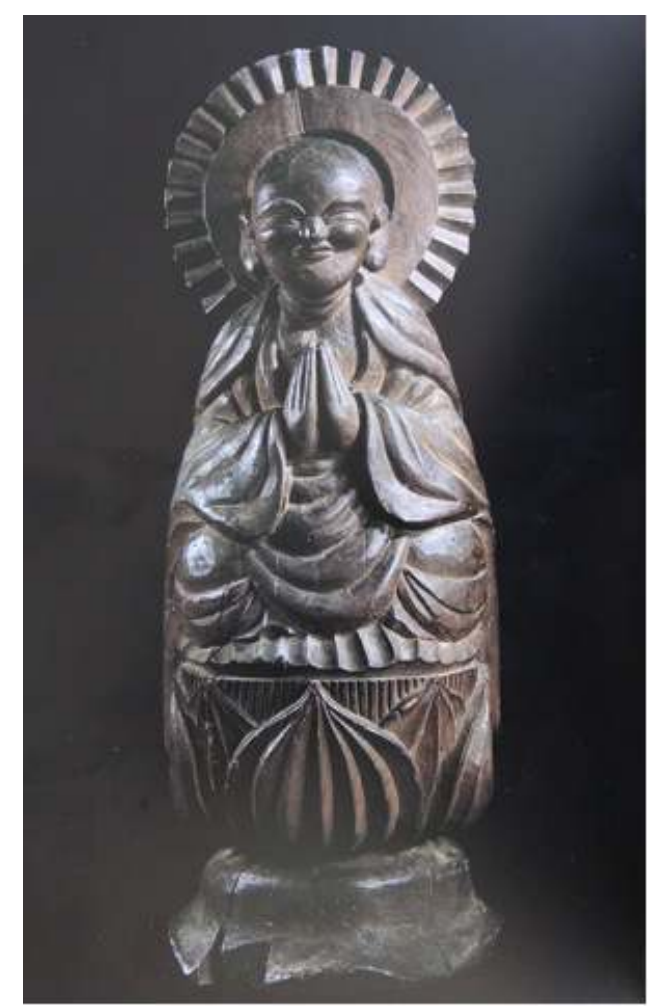

MOKUJIKI, AUTOPORTRAIT À L'ÂgE DE 84 ANS, 1801. MINgEIKAN, TôKYô.

On compte quinze autoportraits dont quatre dans la seule région de Kyōto-Ōsaka. Sur le dos de la statue de l'ermitage d'Inryōan 蔭凉㢆, on peut lire par exemple l'inscription suivante ${ }^{33}$.

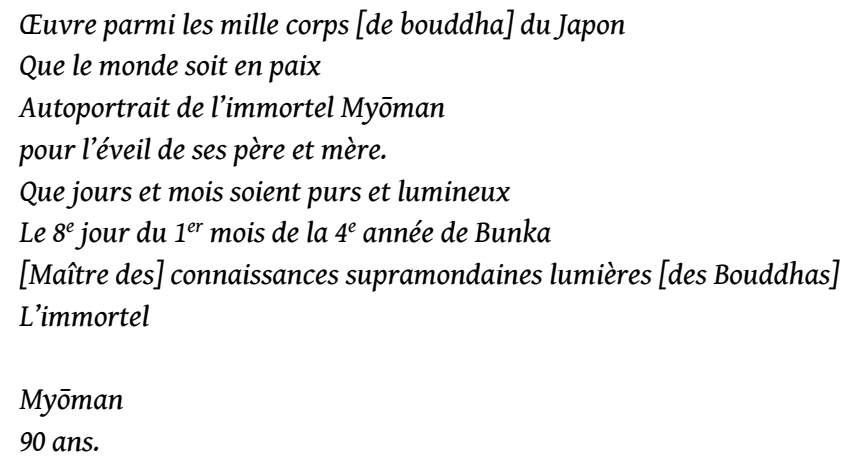

Enkū avait lui aussi réalisé plusieurs "autoportraits». Il est impossible de savoir si ceux-ci, ou ceux de Mokujiki, révèlent des particularités physiques de leurs auteurs. Je pense que c'est probable. Quoi qu'il en soit, si l'on sait que les grands maitres zen donnaient à leur disciple leur propre portrait en guise d'attestation de la transmission, avec les moines vagabonds nous sommes dans un autre registre, celui de l'ascèse qui permet d'accéder à un autre état. Pour Yanagi : 
Le bouddha c'est le Saint homme lui-même. Tous les bouddhas sont des corps de transformation du Saint homme ${ }^{34}$. dans le style de Mokujiki et qui n'appartient à aucune école.

\section{La religion}

31 Le dernier élément qui a rendu possible la découverte de Yanagi se trouve être la religion. Pour Yanagi, il est évident que la foi (il emploie un mot plus souvent traduit par «croyance", shinkō 信仰) transparaît dans les sculptures de Mokujiki. Ces dernières constituent pour lui une oasis, une lumière dans une époque où la religion dépérissait. Yanagi, lui-même, possédait une forte inclination pour la religion. Il resta toute sa vie en contact avec Suzuki Daisetsu 鈴木大拙 (1870-1966), qui avait été son professeur d'anglais au collège de Gakushūin. Celui-ci lui fit mieux connaître le bouddhisme japonais et notamment ses aspects non intellectuels. C'est grâce à ses travaux sur les dévots de la Terre pure, les myōkōnin 妙好人(hommes merveilleusement bons), que Yanagi eut l'idée de faire une étude de terrain dans le département de Tottori $^{35}$. Nous avons vu qu'il s'était intéressé à William Blake dans sa jeunesse. Yanagi a en fait continué à écrire sur des sujets concernant la religion au point de laisser cinq volumes d'œuvres choisies sur ce thème ${ }^{36}$.

La religion de Mokujiki est joyeuse. Les ascèses auxquelles il s'est soumis ne lui ont pas donné une vision pessimiste du corps. Sous son ciseau, tous les êtres semblent saisis par la joie. Même Fudōmyōō 不動明王 (Acalanâtha), Roi de sapience inébranlable, au lieu de montrer son courroux, devient aimable.

Munakata Shikō 棟方志功 (1903-1975), peintre et graveur, ne rencontra Yanagi qu'en 1936 à l'occasion d'une exposition. Il ne fait pas de doute qu'il dut trouver dans l'optimisme des sculptures de Mokujiki une sorte d'écho à ses propres choix esthétiques. On rencontre chez lui les mêmes rondeurs.

\section{La rencontre partagée}

La statue de Jizō que Yanagi rapporta de son voyage à Ikeda ne se situait pas pour lui dans le registre de la belle pièce trouvée par un collectionneur, ni dans celui de l'historien qui trouve un document inconnu. Elle provoque chez lui une émotion esthétique profonde avec de fortes connotations religieuses. C'est son être entier qui s'engage dans une sorte de communion avec Mokujiki, ce moine vagabond d'une extraordinaire vitalité qui passa toute sa vie au milieu des humbles. Dans le récit de sa découverte, Yanagi passe d'ailleurs plusieurs fois au passif, faisant comprendre que ce n'est pas lui qui reconnut la beauté de la statue, mais que c'est celle-ci qui se fit reconnaître.

Quelle que soit sa sensibilité personnelle, Yanagi n'aurait pu faire partager son enthousiasme sans un terreau propice. Il appartenait au cercle de Shirakaba où les admirateurs de Tolstoï transposèrent au Japon les idées du maître sur la profondeur de la sagesse populaire ${ }^{37}$. Dans un autre horizon, c'est aussi l'époque où Yanagita Kunio 柳 田國男 (1875-1962) est en pleine activité3 ${ }^{38}$. Le «peuple ordinaire» (jōmin 常民) que 
celui-ci s'efforce de sauver de l'oubli, avec certains de ses contemporains, est bien celui dans lequel se fondait Mokujiki.

Il ne faut pourtant pas cantonner la vision de Yanagi à une nostalgie d'une vie campagnarde idéalisée, à un goût du terroir un peu chauvin. D'une part, il insiste sur l'importance de la quête spirituelle. C'est la foi profonde de Mokujiki qui transparaît dans le sourire de ses statues. Je n'aurais pas été étonné s'il avait apprécié Hakuin Ekaku 白隠慧鶴 (1686-1768) et ses bouddhas rieurs ${ }^{39}$. Mais je n'ai pas trouvé trace de son intérêt pour la peinture des moines zen, probablement pas assez populaires à son goût. D'une autre part, Yanagi ne se limita jamais au seul Japon métropolitain. Il fut attiré par Blake, mais aussi par l'artisanat coréen. Ce qui était peu commun à une époque d'oppression et de mépris de la part du colonisateur. Il se battit pour sauver la porte Kwanghwa 光化門du palais royal de Séoul, vouée à la destruction, et ouvrit un musée de l'art populaire coréen à Séoul ${ }^{40}$. Il participe aussi à ce grand mouvement d'intérêt d'une partie des intellectuels pour Okinawa. Ses recherches sur l'artisanat des îles du sud rencontrèrent ainsi celles du maître en teinture Serizawa Keisuke.

Mais surtout, Yanagi affirme avec force que Mokujiki n'appartient pas à son époque, que le temps est venu de le découvrir, que c'est un artiste contemporain. Yanagi n'est pas un homme tourné vers le passé. Son réseau d'amis comprend de nombreux artistes. Nous avons rencontré Kawai Kanjirō, Munakata Shikō, Serizawa Keisuke. On pourrait ajouter Umehara Ryūzaburō 梅原龍三郎 (1888-1986), peintre de style occidental proche du cercle de Shirakaba. Aucun d'entre eux n'est un artiste "régionaliste ». Umehara avait suivi les leçons de Renoir, Munakata fut le premier non européen à être primé à la biennale de Venise pour ses gravures, en 1956, la renommée des œuvres de Serizawa a elle aussi largement dépassé le cadre japonais.

Michael Lucken dans son article souligne la rupture entre la première période de la vie intellectuelle de Yanagi où, avec ses amis, il est fasciné par l'individualisme des artistes occidentaux comme Van Gogh, Renoir, Rodin, et la période "mingei », qui est centrée sur des œuvres anonymes produites par le peuple ordinaire. La découverte de Mokujiki se situe à la charnière. Yanagi s'intéresse déjà à la porcelaine artisanale de Corée, mais n'a pas encore lancé son mouvement pour les Arts populaires. Il reste très sensible à l'originalitée ${ }^{41}$ :

Pour le Saint homme qui était libre, il n'y avait pas d'attachement envers la tradition, il n'y avait pas non plus d'opposition à la tradition [...]. L'originalité, ce n'est pas quelque chose qu'il aurait inventé. Elle est née de lui spontanément [mushin ni 無心に] [...]. Il n'y a pas de trace d'imitation dans ses ceuvres. Il ne cherche pas à conserver une manière, il n'a pas non plus de style habituel. Pour moi, il n'y a pas d'hésitation, il faut le commémorer comme le sculpteur de bouddhas le plus original que le Japon ait enfanté.

Je pense que parmi les éléments qui lui permirent de voir la beauté des sculptures de Mokujiki, il ne faut pas oublier l'apprentissage de l'œil auprès des artistes occidentaux, eux-mêmes en rupture avec le classicisme et l'académisme. Nous avons le témoignage de Munakata Shikō, grand admirateur de Van Gogh depuis sa jeunesse, découvrant des sculptures d'Enkū à Ōmiya en 1960. Après les avoir serrées dans ses bras, les avoir embrassées, il s'exclama : «Enkū, ça doit être le Munakata Shikō de l'ère Genroku. Et puis, Munakata Shikō, ça doit être l'Enkū de l'ère Shōwa $»^{42}$. Le même Munakata avait dit qu'il serait un autre Van Gogh. C'est cette abolition du temps que vécut Yanagi en contemplant le Jizō de Mokujiki. 
40

les éléments étaient réunis pour que Mokujiki sorte de l'ombre, le moindre des mérites de Yanagi fut, comme il le pensait avec modestie, d'avoir été l'instrument de sa révélation. Le travail n'est pas encore achevé car Mokujiki semble, plus qu'Enkū, avoir des difficultés à sortir du Japon ${ }^{43}$. Il doit attendre un nouveau Yanagi ${ }^{44}$.

\section{NOTES}

1. Mokujiki Enkū ryōshōnin isakuten 木喰 · 円空両上人遺作展, Hokkaidō, Esamachi kōminkan 江差 町公民館. Sur la réception d'Enkū, voir Tanahashi Kazuaki 棚橋一晃, Enkū no geijutsu 円空の芸 術, Tōkai daigaku shuppankai 東海大学出版会, 1979. Voir également en français : Anne Bouchy, «Une voie de l'“art premier” dans le Japon du XVII siècle. La statuaire d'Enkū, le pérégrin de l'Essentiel », L'Homme, no 165, 2003, p. 143-172.

2. Mochizuki Shinkyō 望月信亨, Bukkyō daijiten 仏教大辞典, 7 volumes. L'édition revue et augmentée date de 1958.

3. Zoku Nihon kōsōden 続日本高僧伝, ouvrage commencé en 1867 et achevé en 1884.

4. Wakan sansai zue 和漢三才図会, ouvrage de Terajima Ryōan achevé en 1715 et composé sur le modèle du Sancai tuhui 三才図会 chinois.

5. Gorai Shigeru 五来重, Enkū butsu 円空佛, Kyōto, Tankōsha 淡交社, 1977, p. 118.

6. Kinsei kijinden 近世畸人后, ouvrage de Ban Kōkei 伴蒿蹊 (1733-1806) publié à Kyōto en 1790 et qui regroupe une centaine de biographies dont celles de Nakae Tōjū, Kaibara Ekiken, des moines Tōsui, Keichū, etc. Réédition en 1972 dans la collection des livres de poche de l'éditeur Iwanami. François Lachaud traduit le titre par Vies d'excentriques de notre temps dans Le vieil homme qui vendait du thé. Excentricité et retrait du monde dans le Japon du XVIII siècle, Paris, Éditons du Cerf, 2010, p. 50.

7. Dai hyakkajiten 大百科事典, Heibonsha 平凡社, 18 volumes.

8. P. 141 de son journal, cité dans Enkū to Hashimoto Heihachi 円空と橋本平八, sous la direction de Honma Masayoshi 本間正義, Kindai no bijutsu 16 近代の美術 16, Shibundō 至文堂, 1973, p. 18.

9. Enkū butsu to no innen 円空佛との因縁, Mingei, septembre 1959.

10. Mokujiki shōnin 木喰上人 (Le vénérable Mokujiki), Yanagi Sōetsu senshū 柳宗悦選集 (๕euvres choisies de Yanagi Sōetsu), Tōkyō, Shunjūsha 春秋社, vol. 9, 1955, rééd. 1972, 396 p.

11. Kai kokushi 甲斐国志, achevé par Matsudaira Sadayoshi 松平定能 en 1814, est composé de 124 livres. La province de Kai correspond à peu près au département actuel de Yamanashi.

12. Notre sculpteur prit le nom de Mokujiki Gyōdō 木喰行道 (Mangeur d'arbre Voie de l'ascèse) après avoir reçu, en 1762, la " défense de mangeur d'arbre» (mokujikikai 木喰戒). À 76 ans, il le changea en Mokujiki Gogyō bosatsu 木喰五行菩薩 (le Bodhisattva Mangeur d'arbre aux Cinq pratiques). Enfin à 89 ans, il se nomma Mokujiki Myōman sennin 木喰明満仙人 (l'Immortel Mangeur d'arbre Plein de lumière).

13. Sur les excentriques de l'époque d'Edo, voir François Lachaud, op.cit.

14. Gorai Shigeru, Itan no hōrōsha no kanjin to waka 異端の放浪者の钬進と和歌 (Poèmes et quête de vagabonds hérétiques), in Tanahashi Kazuaki, Mokujiki butsu, Kajima kenkyūjo shuppankai, 1973, 213 p., ici p. 122.

15. Ibid., p. 123.

16. Voir à ce propos l'article de Christophe Marquet, dans ce numéro. 
17. Abréviation de Namu daishi henjō kongō 南無大師遍照金剛, appellation de Kōbō daishi 弘法 大師 (Kūkai 空海) utilisée pour l'invoquer dans son mausolée du Mont Kōya.

18. Les détails de la découverte sont donnés dans son ouvrage Mokujiki shōnin, op. cit., p. 4-14. Ceux-ci avaient déjà été explicités sous une forme proche en 1925, dans sa Brève biographie du Vénérable Mokujiki Gogyō (Mokujiki Gogyō shōnin ryakuden 木喰五行上人畧伝), elle même reprise sous le titre de Mokujiki shōnin hakken no engi 木喰上人発見の縁起 (Histoire de la découverte du Vénérable Mokujiki) dans Mokujiki butsu édité par Tanahashi Kazuaki, op. cit., p. 180-199.

19. Le terme pavillon ne doit pas tromper. Il devait s'agir d'un bâtiment très simple, juste assez grand pour abriter les statues. Le nombre de quatre-vingt-huit évoquait les « objets principaux d'adoration " (honzon) de chacune des étapes du pèlerinage de Shikoku. Mokujiki y avait ajouté une statue de Kōbō daishi, un autoportrait et une représentation de Daikokuten (Mahākāla).

20. Tanahashi, Mokujiki butsu, op. cit. p. 98.

21. Il s'agit des Dix grands vœux du bodhisattva Samantabhadra (Fugen bosatsu 普賢菩薩), tels que rapportés dans le Sūtra de l'Ornementation fleurie (Kegonkyō 華誉経), et exprimant la compassion du bodhisattva envers les êtres vivant en ce monde.

22. Yadochō 宿帳: carnets dans lesquels Mokujiki notait chaque jour le nom du village ou du hameau où il se trouvait et le type d'hébergement, temple, chapelle, ou le nom de la personne qui le recevait. Presque tous les toponymes étaient notés en katakana.

23. Hōkyōchō 奉經帳: carnets dans lesquels étaient recensés les objets que Mokujiki avait exécutés et déposés en offrande.

24. Ainsi, c'est parce qu'il savait par les papiers de Mokujiki que celui-ci avait séjourné longuement à Sado qu'il s'y rendit lui-même.

25. Mushanokōji était son aîné au collège de Gakushūin, établissement fréquenté par les enfants de la maison impériale, de l'aristocratie et de la haute société, membre du groupe tolstoïen Shirakaba tout comme un autre ami de Yanagi, Shiga Naoya 志賀直哉 (1883-1971).

26. Il donnera l'édition définitive en 1955, avec des vignettes de Serizawa Keisuke, dans le volume 9 de ses ๔uvres choisies publiées chez Shunjūsha. Serizawa Keisuke 芹沢銈介 (1895-1984), graveur et maître en teinture, entra en contact avec Yanagi en 1928 après avoir lu son essai Kōgei no michi 工藝の道 (La voie de l'artisanat).

27. Hachijūhachi-tai butsu no bi八十八射佛の美 (La beauté des 88 corps de bouddha), in Mokujiki shōnin , op. cit., p. 237-245, notamment p. 243 sqq.

28. Yanagi insiste sur la difficulté de retrouver les noms de lieux inscrits sur les carnets d'hébergement. Beaucoup ne figurent sur aucune carte.

29. Ibid., p. 244.

30. Ce fut aussi le point de vue de Kawai Kanjirō 河井寛次郎 (1890-1966), sculpteur mais surtout potier, qui s'était fait un nom dès 1921 grâce à une exposition aux grands magasins Takashimaya. Kawai ne parvenait pas à être satisfait de son travail. Attiré par la porcelaine de la dynastie Yi qu'étudiait Yanagi, il partagea l'enthousiasme de ce dernier pour les objets anonymes de l'artisanat et l'accompagna dans plusieurs de ses enquêtes de terrain à la recherche des sculptures de Mokujiki. Sur les Ōtsu-e, se reporter à l'article de Christophe Marquet.

31. Shuji 種子: une lettre de l'alphabet sanscrit est mise en correspondance avec un bouddha ou un bodhisattva et sert à le représenter ou l'invoquer. Par exemple la lettre kiriku (hrîh) pour Amida.

32. Mokujiki shōnin, op. cit., p. 114-115.

33. Tanahashi, Mokujiki butsu, op. cit., p. 133.

34. «Butsu wa shōnin mizukara de aru»佛は上人自らである; Hachijūhachi-tai butsu no bi, in Mokujiki shōnin, op. cit., p. 244-245.

35. Il en publia le résultat sous le titre de Genza d'Inaba, Homme merveilleusement bon (Myōkōnin Inaba no Genza 妙好人因幡源左). Genza d'Inaba (1842-1930) était un simple paysan connu pour la 
profondeur de sa foi en Amida. La monographie signée de Yanagi et de Kinugasa Isshō 衣笠一省 fut publiée en 1960 chez Hyakkaen 百華苑.

36. Yanagi Muneyoshi shūkyō senshū 柳宗悦・宗教選集 (Tōkyō, Shunjūsha, 1960-1961) : 1. Shūkyō to sono shinri 宗教とその真理 (La religion et sa vérité), 2. Shūkyō no rikai 宗教の理解 (La compréhension de la religion), 3. Kami ni tsuite 神について (À propos du divin), 4. Namu Amidabutsu 南無阿弥陀佛 (Invocation au Bouddha Amida), Ippen shōnin一遍上人 (Le saint homme Ippen), 5. Shūkyō zuisō 宗教随想 (Réflexions sur la religion).

37. Voir dans ce même numéro l'article de Michael Lucken.

38. Voir l'article de Damien Kunik et la traduction de l'entretien des deux hommes.

39. Voir par exemple Hakuin zen to shoga. Hakuin zenji seitan 320 nen 白隠禅と書画一白隠禅師生 誕320年 (Le zen de Hakuin, peintures et calligraphies. $320^{\mathrm{e}}$ anniversaire de la naissance du maitre de méditation Hakuin), Kyōto bunka hakubutsukan 京都文化博物館, 2004.

40. Voir, dans un prochain numéro de Cipango consacré à la Corée et à l'époque coloniale, l'article de Pierre Souyri : « La critique du colonialisme dans le Japon d'avant-guerre ».

41. Hachijūhachi-tai butsu no bi, in Mokujiki shōnin, op. cit., p. 237.

42. Akiyama Kikuo 秋山喜久夫, Koi Enkū 恋円空, Urawa, Saitama ken kyōdo shiryō kankōkai 埼 玉県郷土史料刊行会, 1973, p. 23.

43. À ma connaissance, il n'y a pas encore eu d'exposition consacrée à Mokujiki en Europe, alors qu'Enkū a été exposé à Anvers en 1999. Voir Jan Van Alphen, Robert Duquenne et al., Enkū, 1632-1695: Timeless Images from 17th Century Japan, Anvers, Etnografisch Museum.

44. Je remercie Christophe Marquet et Jean-Michel Butel pour leur relecture attentive et leurs corrections.

\section{RÉSUMÉS}

Au début des années 1920, Yanagi Sōetsu découvre par hasard les œuvres d'un sculpteur tombé dans l'oubli : Mokujiki (1718-1810). Il va dès lors fortement contribuer à sa reconnaissance.

At the start of the 1920s, Yanagi Sōetsu stumbled upon Buddhist sculptures of an almost unknown artist: Mokujiki (1718-1810). He then began to increase public awareness of his work. 
INDEX

Mots-clés : mingei - mouvement artistique, culture populaire, arts populaires, Yanagi Sōetsu (1889-1961), Mokujiki, Enkū, bouddhisme, sculpture bouddhique, moine vagabond

Thèmes : ethnologie, anthropologie, sciences des religions

キーワード : minshū geijutsu 民衆芸術, bukkyō 仏教, taishū bunka 大衆文化, Enkū 円空

(1632-1695), mingei undō 民芸運動, yugyōsō 遊行層, bukkyō chōkoku 仏教彫刻, Mokujiki 木喰 (1718-1810), Yanagi Sōetsu 柳宗悦 (1889-1961), jinruigaku 人類学, minzokugaku 民族学, shūkyōgaku 宗教学, Edo jidai 江戸時代 (1603-1868), Taishō jidai 大正時代 (1912-1923), Shōwa jidai 昭和時代 (1923-1945), Shōwa jidai 昭和時代 (1945-1989)

Keywords : Utensils, Material Culture, Folk Art, Popular Culture, Mingei, Yanagi Sōetsu (1889-1961), Anthropology, Ethnology, Mokujiki, Buddhism, Buddhist Sculpture, Folklore, Enkū, Itinerant Monk

Index chronologique : Shōwa (1923-1945), Shōwa (1945-1989) 